\title{
COMPLETELY RIGHT INJECTIVE SEMIGROUPS THAT ARE UNIONS OF GROUPS $†$
}

\author{
by E. H. FELLER and R. L. GANTOS
}

(Received 16 July, 1969; revised 16 July, 1970)

1. Introduction. A semigroup $S$ with 0 and 1 is termed completely right injective provided every right unitary $S$-system is injective. A necessary condition for a semigroup to be completely right injective is given in [2]; namely, every right ideal is generated by an idempotent. An example in section 3 of this paper shows the existence of semigroups with 0 and 1 satisfying this condition which are not completely right injective. In [3], it is shown that the condition that every right and left ideal is generated by an idempotent is necessary and sufficient in the case that $S$ is both completely right and left injective (called completely injective). Such a semigroup is an inverse semigroup with 0 whose idempotents are dually well-ordered.

The purpose of this paper is to give a characterization for semigroups which are completely right injective and a union of groups and to determine a decomposition for such semigroups. We first develop several properties concerning the two-sided ideals of a semigroup which satisfies the condition that every right ideal is generated by an idempotent. We give equivalent conditions for semigroups of this type to be a union of groups. Using these properties, we are able to prove the characterization. The main theorem states that a semigroup $S$ is completely right injective and is a union of groups if and only if every right ideal I of $S$ is generated by an idempotent which commutes with all the elements of $S$ not in $I$. It is shown that a semigroup of this type is a chain of right groups. In addition, all completely right injective semigroups which have a finite number of right ideals are unions of groups.

We follow the definitions and notations introduced in [2] and [3] and use freely the results proved there; otherwise the notation and terminology is that of Clifford and Preston [1]. Throughout this paper all semigroups will have 0 and 1 and all $S$-systems will be right unitary $S$-systems.

2. Completely right injective semigroups. In this section, with the exceptions of Theorems $2.10,2.11$, and $2.12, S$ will always denote a semigroup with 0 and 1 such that every right ideal is generated by an idempotent. In the aforementioned theorems, $S$ will denote a completely right injective semigroup. As in [3], the lattice of right ideals of $S$ under set inclusion is dually wellordered. In addition, $S$ is a regular semigroup [1, p. 27]. An inverse of an element $s$ in $S$ will usually be denoted by $s^{\prime}$, i.e., $s=s s^{\prime} s$ and $s^{\prime}=s^{\prime} s s^{\prime}$, although $s^{\prime}$ need not be unique. Consequently, if $s \in S$ and $s S=e S$ for some $e \in E(S)$, where $E(S)$ denotes the subsemigroup of all idempotents in $S$, there exists an inverse $s^{\prime}$ of $s$ such that $s s^{\prime}=e$. Moreover, $s S=s s^{\prime} S$ and $S s=S s^{\prime} s$.

Since the right ideals of $S$ are linearly ordered we have

2.1. Proposition. If $S e=S f$, for $e, f \in E(S)$, then $e=f$.

2.2. Proposition. If $e \in E(S), s \in S$, then Ses $=S s^{\prime} e s$.

$\dagger$ Research supported by the National Science Foundation under grant GP-6816 for the first author. 
Proof. We need only show that $S s^{\prime} e s$ contains es. If $s S \supseteq e S$, then $s\left(s^{\prime} e s\right)=\left(s s^{\prime}\right) e s=e s$. If $s S \subseteq e S$, then $e s=s$ and $e s\left(s^{\prime} e s\right)=e s$.

For each $e \in E(S)$, we have $s^{\prime} e s \in E(S)$. Consequently, 2.1 and 2.2 imply

2.3. Proposition. If $s^{\prime}$ and $s^{\prime \prime}$ are inverses of an element $s$ in $S$, then $s^{\prime} e s=s^{\prime \prime} e s$.

As defined in [1, pp. 47-48], $\mathscr{H}, \mathscr{R}$ and $\mathscr{L}, \mathscr{J}$ will denote Green's equivalence relations on the semigroup $S . \quad L_{a}\left[R_{a}, H_{a}\right]$ denotes the $\mathscr{L}$-[R्R-, $\left.\mathscr{H}-\right]$ class of $S$ containing the element $a$.

2.4. Proposition. Each $\mathscr{L}$-class of $S$ contains exactly one idempotent.

Proof. Since $S$ is regular, every $\mathscr{L}$-class contains an idempotent. By 2.1 , it is unique.

The following proposition is true for any regular semigroup.

2.5. PROPOSITION. If $x s S=s x S$, where $x$ is an inverse of $s$, then there exists an inverse $s^{\prime}$ of $s$ such that $s^{\prime} s=s^{\prime}$.

Proof. Now $x s S=s x S$ implies that $(s x)(x s)=x s$ and $x s s=s$. Set $s^{\prime}=x^{2} s$. Then

$$
\begin{aligned}
s s^{\prime} s & =(s x)(x s s)=s x s=s \\
s^{\prime} s s^{\prime} & =x(x s s)\left(x^{2} s\right)=(x s x)(x s)=x^{2} s=s^{\prime} \\
s s^{\prime} & =(s x)(x s)=x s=x(x s s)=\left(x^{2} s\right) s=s^{\prime} s .
\end{aligned}
$$

2.6. Proposition. a $\mathscr{L} b$ implies $a^{\prime} \mathscr{R} b^{\prime}$ for all $a, b \in S$.

Proof. Now $a \mathscr{L} b$ implies $S a^{\prime} a=S b^{\prime} b$. By 2.1 we have $a^{\prime} a=b^{\prime} b$. Thus $a^{\prime}=a^{\prime} a a^{\prime}=b^{\prime} b a$ and $a^{\prime} S \subseteq b^{\prime} S$. Similarly, $b^{\prime} S \subseteq a^{\prime} S$.

The following results give some special properties concerning (two-sided) ideals of $S$.

2.7. Proposition. Let I be an ideal of $S$ and $a, b, c \in S$.

(i) If $a \in I$, then every inverse $a^{\prime}$ of $a$ is in $I$.

(ii) If $a \notin I$ and $c \in I$, then $S a c=S c$.

(iii) $I$ is a prime ideal of $S$. [2, p. 40].

(iv) The relation $\rho$, defined by $a \rho b$ if and only if either $a, b \in I$ and $a \mathscr{L} b$ or $a, b \notin I$, is a right congruence on $S$.

Proof. The first part follows from the fact that $a^{\prime}=a^{\prime} a a^{\prime}$ and $I$ is an ideal of $S$. Now $a \notin I$ implies $a^{\prime} a \notin I$. If $c \in I$, then we have $c S \subseteq a^{\prime} a S$ so that $a^{\prime} a c=c$. This proves (ii). Moreover, either $a^{\prime} a S \subseteq c c^{\prime} S$ or $c c^{\prime} S \subseteq a^{\prime} a S$. The former implies $a=a\left(c c^{\prime}\right)\left(a^{\prime} a\right)$ and the latter $c=\left(a^{\prime} a\right)\left(c c^{\prime}\right) c$. Consequently, $a c \in I$ implies either $a \in I$ or $c \in I$. This completes the proof of (iii).

The relation $\rho$ defined in (iv) is clearly an equivalence relation on $S$. Suppose $a \rho b$ and $c \in S$. Since $\mathscr{L}$ is a right congruence on $S$ we may assume $a, b \notin I$. If $c \in I$, then, by (ii), $S a c=$ $S c=S b c$. If $c \notin I$, then (iii) implies that $a c$ and $b c$ are not elements of $I$. In either case we have $a c \rho b c$. 
Let $D(S)$ denote the subset of $E(S)$ consisting of all elements which generate the (twosided) ideals of $S$. Since the collection $\mathfrak{I}(S)$ of all ideals of $S$ is a dually well-ordered set with respect to set inclusion, then we can write the chain of all ideals in the following manner.

$$
S=d_{0} S \supset d_{1} S \supset d_{2} S \supset \ldots \supset d_{\alpha} S \supset \ldots,
$$

where the subscripts belong to the set $M_{\gamma}$ of all ordinals less than the ordinal $\gamma$ of the dual of $\mathfrak{I}(S)$, and $d_{\alpha} \in D(S)$.

2.9. Proposition. For each ordinal $\alpha$ in $M_{\gamma}$, let us define $T_{\alpha}=d_{\alpha} S \backslash d_{\alpha+1} S$. Then $T_{\alpha}$ is a subsemigroup of $S$ for which $a \in T_{\alpha}$ implies that $a^{\prime} \in T_{\alpha}$, where $a^{\prime}$ is any inverse of $a$. Moreover $\left\{T_{\alpha} \mid \alpha \in M_{\gamma}\right\}$ is the set of all $\mathscr{J}$-classes of $S$.

Proof. Applying 2.7 (iii), one can easily show that $T_{\alpha}$ is a subsemigroup of $S$. Let $a \in T_{\alpha}$. Since $a^{\prime}=a^{\prime} a a^{\prime}, a \in d_{\alpha} S$, and $d_{\alpha} S$ is an ideal of $S$, it follows that $a^{\prime} \in d_{\alpha} S$. On the other hand, since $a=a a^{\prime} a, a \notin d_{\alpha+1} S$ and $d_{\alpha+1} S$ is an ideal of $S$, we must have that $a^{\prime} \notin d_{\alpha+1} S$. Hence $a \in T_{\alpha}$ implies that $a^{\prime} \in T_{\alpha}$.

Let $\alpha \in M_{\gamma}$. We show that $T_{\alpha}$ is precisely the $\mathscr{J}$-class of $S$ containing the idempotent $d_{\alpha}$. Let $a \in T_{a}$. Then $S a S \subseteq S d_{\alpha} S=d_{\alpha} S$. Since the ideals of $S$ are linearly ordered and $a \notin d_{\alpha+1} S$, it follows that $d_{\alpha+1} S=S d_{\alpha+1} S \subset S a S$. Therefore $d_{\alpha+1} S \subset S a S \subseteq d_{\alpha} S$, and because $d_{\alpha+1} S$ is the maximal ideal of $S$ contained in $d_{\alpha} S$, this implies that $S a S=d_{\alpha} S$. Thus $a \mathscr{f} d_{\alpha}$. On the other hand, if $b$ is an element of $S$ for which $b \mathscr{J} d_{\alpha}$, then $S b S=d_{\alpha} S$ which, in turn, implies that $b \in T_{a}$.

Since each element of $S$ belongs to some $T_{\alpha}$, then the above implies that each $\mathscr{E}$-class of $S$ coincides with some $T_{\alpha}$. Thus the set, $\left\{T_{\alpha} \mid \alpha \in M_{\gamma}\right\}$, is the set of all $\mathscr{J}$-classes of $S$.

2.10. TheOREM. Let $S$ be a completely right injective semigroup and let I be an ideal of $S$. There exists an idempotent $d \in S$ such that $I=d S$, and $d s=s d$ for all $s \notin I$.

Proof. If $I=S$, the statement is trivially true. Thus we assume that $I$ is a proper ideal of $S$. Let $\rho$ be the right congruence on $S$ defined in 2.7 (iv). We consider the right $S$-system $S / \rho$ consisting of all the $\rho$-classes of $S$, where the system product is given by $(x \rho) s=(x s) \rho$, $x \rho \in S / \rho$ and $s \in S$. Let $N=\{x \rho \mid x \in I\}$. Since $I$ is an ideal, $N$ is an $S$-subsystem of $S / \rho$. Also we note that $x \rho \subseteq I$ if $x \in I$.

Since $S$ is completely right injective, the identity mapping $1_{N}: N \rightarrow N$ can be extended to an $S$-homomorphism $\pi: S / \rho \rightarrow N$. By 2.4, if an equivalence class $x \rho$ is in $N$, then it contains one and only one idempotent; namely, the idempotent $x^{\prime} x$. Consequently, we can write $\pi(1 \rho)=d \rho$, where $d$ is an idempotent in $I$. If $I=e S$, where $e \in E(S)$, then $d S \subseteq e S$. However,

$$
e \rho=1_{N}(e \rho)=\pi(e \rho)=\pi(1 \rho) e=(d \rho) e=(d e) \rho .
$$

Thus $e=d e$, and it follows that $d S=e S=I$.

Let $s \notin I$. Then $\pi(1 \rho)=\pi(s \rho)=\pi(1 \rho) s=(d s) \rho$. By 2.2, we have $(d s) \rho=\left(s^{\prime} d s\right) \rho$. Therefore $d \rho=\left(s^{\prime} d s\right) \rho$ which, in turn, implies $d=s^{\prime} d s$. Since $s \notin I$, then $s s^{\prime} \notin I$, and we have $s d=$ $s\left(s^{\prime} d s\right)=d s$. 
2.11. Proposition. Let $S$ be a completely right injective semigroup and let $I$ be an ideal of $S$. Then $K$ is a left [right, two-sided] ideal of I if and only if $K$ is a left [right, two-sided] ideal of $S$ contained in $I$.

Proof. Assume $K$ is a left ideal of $I$. Let $s \in S, s \notin K$ and $k \in K$. If $s \in I$, then $s k \in K$, for $K$ is a left ideal of $I$. If $s \notin I$, then $s k=s(d k)=(s d) k=(d s) k \in K$, where $d$ is the idempotent, defined in 2.10, which generates $I$.

Suppose $K$ is a right ideal of $I$. Let $s \in S, s \notin K$ and $k \in K$. Now $k \in K$ implies $k^{\prime} k \in I$ which, in turn, gives $d k^{\prime} k=k^{\prime} k$. Hence $k s=k\left(d k^{\prime} k s\right) \in K I$. Since $K I \subseteq K$, we have $k s \in K$.

2.12. Proposition. If $S$ is completely right injective, then the semigroups $T_{\alpha}(\alpha<\gamma)$ of 2.9 are simple.

Proof. Let $K \neq \emptyset$ be a (two-sided) ideal of $T_{\alpha}$. Then $K \cup d_{\alpha+1} S$ is an ideal of $d_{\alpha} S$. By 2.11, $K \cup d_{\alpha+1} S$ is an ideal of $S$ and $d_{\alpha+1} S \subset K \cup d_{\alpha+1} S \subseteq d_{\alpha} S$. It follows that $K \cup d_{\alpha+1} S=d_{\alpha} S$ which, in turn, implies $K=T_{\alpha}$.

3. Completely right injective semigroups that are unions of groups. We begin with a theorem which does not require the injective property.

3.1. THEOREM. Let $S$ be a semigroup with 0 and 1 such that every right ideal is generated by an idempotent. Then the following are equivalent.

(i) $S$ is the union of groups.

(ii) Every $\mathscr{L}$-class of $S$ is a group.

(iii) Every right ideal of $S$ is two-sided.

Proof. (i) implies (ii). Since $S$ is a union of groups, each $\mathscr{H}$-class of $S$ is a group [1, Theorem 4.3]. We will have (ii) provided we show that $\mathscr{H}=\mathscr{L}$. Suppose $a \mathscr{L} b$. Then $a, b \in L_{e}$, where, according to $2.4, e$ is the unique idempotent belonging to $L_{c}$. Since $H_{a} \subseteq L_{e}$, $H_{b} \subseteq L_{e}$, and since both $H_{a}$ and $H_{b}$ contain idempotents, we have $e \in H_{a} \cap H_{b}$. Hence $H_{a}=H_{b}$ so that $a \mathscr{H} b$. This proves (ii). Since $S$ is a union of its $\mathscr{L}$-classes, (ii) implies (i).

(ii) implies (iii). Let $e S$, where $e \in E(S)$, be a right ideal of $S$. Let $a \in e S$ and $s \in S$. We want to show $s a \in e S$. Since $e S$ is a subsemigroup, we may assume that $s \notin e S$. This implies that $a S \subset e S \subset s S$. Since $S$ is a union of its $\mathscr{L}$-classes, $s \in L_{f}$ for some $f \in E(S)$. Because $L_{f}$ is a group with identity $f$, there exists $t \in L_{f}$ such that $t s=f$. From $a S \subset s S=f S$ we conclude that $a=f a$. Therefore $a=f a=(t s) a=t(s a) \in S s a$. This implies that $S a=S s a$ and hence $s a \in L_{a}$. Since $L_{a}$ is a group, there exists $u \in L_{a}$ such that $s a=a u$. Thus $s a \in e S$.

(iii) implies (ii). Let $L_{e}$ be an $\mathscr{L}$-class of $S$, where $e$ is the unique idempotent of $S$ contained in $L_{e}$. We show $L_{e}=H_{e}$ which, together with Theorem 2.16 of [1], implies that $L_{e}$ is a group. By 2.6, $a \mathscr{L} e$ implies that $a^{\prime} \mathscr{R} e$, where $a^{\prime}$ is any inverse of $a$. However, $a^{\prime} S$ is a two-sided ideal of $S$, so that $a=a a^{\prime} a \in a^{\prime} S=e S$. Hence $a S \subseteq e S$. On the other hand, from $a^{\prime} a \in L_{e}$ and 2.4 we can conclude that $a^{\prime} a=e$. Since $a S$ is a two-sided ideal of $S, e=a^{\prime} a \in a S$ so that $e S \subseteq a S$. Therefore $a S=e S$ and $a \in R_{e}$. Hence $L_{e} \subseteq R_{e}$ from which we conclude that $L_{e}=H_{e}$. 
3.2. MAIN THEOREM. Let $S$ be a semigroup with 0 and 1. Then $S$ is completely right injective and a union of groups if and only if every right ideal $I$ is generated by an idempotent $d$ such that $d s=s d$ for all $s \notin I$.

Proof. The necessity follows from 3.1 and 2.10 .

Assume that the right ideals of $S$ satisfy the condition in the statement of the theorem. We first prove that every right ideal of $S$ is two-sided. It then follows, by 3.1 , that $S$ is a union of groups. Let $I$ be a right ideal of $S$. It suffices to show that $s a \in I$ for all $a \in I$ and $s \in S \backslash I$. Since $s \notin I$, our assumption implies that $s a=s(d a)=(s d) a=(d s) a \in I$.

To show that $S$ is completely right injective we use the technique employed in the proof of 2.6 of [2]. Let $M, P$, and $R$ be $S$-systems, where $P \subseteq R$, and let $f: P \rightarrow M$ be an $S$-homomorphism of $P$ into $M$. As in [2, 2.6], we can use Zorn's Lemma to obtain a maximal pair $\left(P_{0}, f_{0}\right)$ consisting of a subsystem $P_{0}$ of $R$, where $P_{0} \supseteq P$, and an $S$-homomorphism $f_{0}: P_{0} \rightarrow M$, where $f_{0}$ extends $f$. To show that $M$ is injective it suffices to show $P_{0}=R$.

Suppose that $P_{0} \subset R$ and let $r \in R$ be such that $r \notin P_{0}$. Set $A=\left\{a \in S \mid r a \in P_{0}\right\}$. In the two cases, $A$ non-empty or $A$ empty, we will be able to define an $S$-homomorphism $h$ of $r S$ into $M$ which agrees with $f_{0}$ on $P_{0} \cap r S$.

If $A$ is empty, define $h: r S \rightarrow M$ by $h(x)=m 0$ for all $x \in r S$, where $m$ is an arbitrary but fixed element of $M$. Then $P_{0} \cap r S$ is empty and $h(x) s=(m 0) s=m 0=h(x s)$ for all $x \in r S$ and $s \in S$. Thus $h$ is an $S$-homomorphism of $r S$ into $M$.

Suppose that $A$ is non-empty. Then $A$ is a right ideal of $S$ and hence by hypothesis, $A=d S$, where $d$ is an idempotent of $S$ such that $s d=d s$ for all $s \notin A$. Define $h$ by $h(r s)=f_{0}(r d s)$ for all $s \in S$. From the definition of the set $A$ we conclude that $h(r s) \in M$ for all $s \in S$. First of all, we have that $r s_{1}=r s_{2}$, where $s_{1}, s_{2} \in S$, implies that $r d s_{1}=r d s_{2}$. Indeed, the definition of the set $A$ yields that both $s_{1}$ and $s_{2}$ either are or are not members of $A$. In either situation we conclude that $r d s_{1}=r d s_{2}$; the latter uses the fact that $s_{1}$ and $s_{2}$ commute with $d$. This together with the single-valued property of $f_{0}$ implies that

$$
h\left(r s_{1}\right)=f_{0}\left(r d s_{1}\right)=f_{0}\left(r d s_{2}\right)=h\left(r s_{2}\right)
$$

Hence $h: r S \rightarrow M$ is a map of $r S$ into $M$. Since $f_{0}$ is an $S$-homomorphism, then $h$ is an $S$-homomorphism. Also if $x \in P_{0} \cap r S$, then $x=r a \in P_{0}$, where $a \in A$. Since $d a=a$, then

$$
h(x)=h(r a)=f_{0}(r d a)=f_{0}(r a)=f_{0}(x)
$$

Thus $h$ is an $S$-homomorphism of $r S$ into $M$ which agrees with $f_{0}$ on $P_{0} \cap r S$.

Set $P^{*}=P_{0} \cup r S$ and let $f^{*}: P^{*} \rightarrow M$ be the map defined by $f^{*}(x)=f_{0}(x)$, if $x \in P_{0}$, and $f^{*}(x)=h(x)$, if $x \in r S$, where $h(x)$ is the map defined above, according to the appropriate case where $A$ is empty or non-empty. It follows that $f^{*}$ is an $S$-homomorphism of $P$ into $M$ which extends $f_{0}$. Hence $\left(P^{*}, f^{*}\right)>\left(P_{0}, f_{0}\right)$, which contradicts the maximality of the pair $\left(P_{0}, f_{0}\right)$. Thus $P_{0}=R$ and $M$ is injective.

Let $S$ be a completely right injective semigroup which is a union of groups. By applying 
(2.8), the chain of all right (and hence two-sided) ideals of $S$ can be exhibited in the following manner.

$$
S=d_{0} S \supset d_{1} S \supset d_{2} S \supset \ldots \supset d_{\alpha} S \supset \ldots,
$$

where $\alpha \in M_{y}$ and, by 3.1 (iii), $d_{\alpha}$ is an idempotent of $S$ which commutes with all elements of $S$ not in $d_{\alpha} S$.

3.4. THEOREM. Let $S$ be a completely right injective semigroup which is a union of groups. Then $T_{\alpha}=d_{\alpha} S \backslash d_{\alpha+1} S(\alpha<\gamma)$, is a right group. In addition, $S$ is a chain $M_{\gamma}$ of right groups $T_{\alpha}\left(\alpha \in M_{y}\right)$.

Proof. Let $a \in T_{\alpha}$. Since $d_{\alpha+1} S$ is the maximal right ideal of $S$ contained in $d_{\alpha} S$, we must have $d_{\alpha} S=a S$. Hence there exists an inverse $a^{\prime}$ of $a$ such that $a a^{\prime}=d_{\alpha}$. Since $d_{\alpha} S$ and $d_{\alpha+1} S$ are two-sided ideals and since $a=a a^{\prime} a$ and $a^{\prime}=a^{\prime} a a^{\prime}$, it follows that $a^{\prime} \in T_{\alpha}$. If $b \in T_{\alpha}$, then $b=d_{\alpha} b=a a^{\prime} b$. By $2.9, T_{\alpha}$ is a subsemigroup of $S$. Thus $a^{\prime} b \in T_{\alpha}$ so that $b \in a T_{\alpha}$. This proves that $T_{\alpha}=a T_{\alpha}$ for all $a \in T_{\alpha}$. Therefore $T_{\alpha}$ is right simple and contains an idempotent. Applying Theorem 1.27 (ii) of $\left[1\right.$, p. 38], we have that $T_{\alpha}$ is a right group.

Clearly $S$ is the disjoint union of right groups $T_{\alpha}\left(\alpha \in M_{\gamma}\right)$. Following the terminology of $\left[1\right.$, p. 25], we will have that $S$ is a chain $M_{\gamma}$ of right groups $T_{\alpha}\left(\alpha \in M_{\gamma}\right)$ if we can show that $T_{\alpha} T_{\beta} \subseteq T_{\beta}$ and $T_{\beta} T_{\alpha} \subseteq T_{\beta}$ for all $\alpha, \beta \in M_{\gamma}$, where $\alpha<\beta$. Let $\alpha, \beta \in M_{\gamma}$, where $\alpha<\beta, a \in T_{\alpha}$ and $b \in T_{\beta}$. We have that $d_{\beta+1} S \subset d_{\beta} S \subseteq d_{\alpha+1} S \subset d_{\alpha} S$. Since $d_{\beta} S$ is two-sided and $b \in d_{\beta} S$, it follows that $a b$ and $b a$ are elements in $d_{\beta} S$. By 2.9, we have that $a, a^{\prime}, a^{\prime} a$ and $a a^{\prime}$ all belong to $T_{\alpha}$. Consequently, $a S=a^{\prime} S=a a^{\prime} S=a^{\prime} a S=d_{\alpha} S$. Likewise, $b S=b^{\prime} S=b b^{\prime} S=b^{\prime} b S=$ $d_{\beta} S$. Because $b S \subset a^{\prime} a S$, it follows that $b=a^{\prime} a b$. In addition, since $b^{\prime} b S \subset a a^{\prime} S$, we have that $b^{\prime} b=a a^{\prime} b^{\prime} b$ which, in turn, implies that $b=b a a^{\prime} b^{\prime} b$. The expression $b=a^{\prime} a b=b a a^{\prime} b^{\prime} b$ together with the fact that $d_{\beta+1} S$ is two-sided implies that neither $a b$ nor $b a$ belongs to $d_{\beta+1} S$; for otherwise, in both cases, we will have that $b \in d_{\beta+1} S$, which is not true. Thus $a b$ and $b a$ belong to $T_{\beta}$.

Using known properties of right groups, we can apply 3.4 to give additional properties of a semigroup $S$ which is completely right injective and a union of groups. Because of Theorem 1.27 (iii) of [1, p. 38], each of the right groups $T_{\alpha}(\alpha<\gamma)$ is the direct product of a group $G_{\alpha}$ and a right zero semigroup $E_{\alpha}$. In addition, Problem 3 of [1, p. 39] implies that $T_{\alpha}$ is the union of isomorphic disjoint groups; namely $T_{\alpha}=\bigcup L_{g}$, where the union ranges over all idempotents $g$ in $T_{\alpha}$. This reminds one of the decomposition of semi-simple rings.

3.5. THEOREM. If $S$ is completely right injective and has a finite number of right ideals, then $S$ is a union of groups.

Proof. Let $a \in S$ and let $a^{\prime}$ be an inverse of $a$. The mapping $h: a^{\prime} a S \rightarrow a S\left(=a a^{\prime} S\right)$ defined by $h\left(a^{\prime} a s\right)=a s$, for all $s \in S$, is an $S$-isomorphism of the $S$-subsystem $a^{\prime} a S$ onto the $S$ subsystem $a a^{\prime} S$. This $S$-isomorphism requires that the number of right ideals in the chain of all right ideals of $S$ contained in $a^{\prime} a S$ equals the number in the chain of all right ideals of $S$ contained in $a a^{\prime} S$. Hence we cannot have either $a a^{\prime} S \subset a^{\prime} a S$ or $a a^{\prime} S \supset a^{\prime} a S$. That is, $a a^{\prime} S=a^{\prime} a S$ and from 2.5 we conclude that $a a^{\prime \prime}=a^{\prime \prime} a$ for some inverse $a^{\prime \prime}$ of $a$. Since $a \mathscr{R} a a^{\prime \prime}$ 
and $a^{\prime \prime} a \mathscr{L} a$, this implies that $a \mathscr{H} a a^{\prime \prime}$. Hence $H_{a}$ contains an idempotent and, by Theorem 2.16 of $\left[1\right.$, p. 59], $H_{a}$ is a group. Since $S$ is the union of its $\mathscr{H}$-classes we have our result.

In view of 3.5 and the obvious fact that an idempotent semigroup is a union of groups we can apply the main theorem to prove the following result.

3.6. THEOREM. A semigroup with 0 and 1 which is either idempotent or contains a finite number of right ideals is completely right injective if and only if each right ideal I of $S$ contains an idempotent generator which commutes with all elements not in $I$.

An example of an idempotent semigroup which is completely right injective can be constructed as follows.

Let $E$ and $F$ be two disjoint right zero semigroups. Define $e f=f e=e$ for all $e \in E$ and $f \in F$. This product together with the product already defined in $E$ and $F$ make $E \cup F$ into a semigroup. If we adjoin 0 and 1 to $E \cup F$, then the resultant semigroup is completely right injective. Also $T=E \cup F \cup 0 \cup 1$ can be made into a completely right injective semigroup by defining $f e=e$ and $e f=e^{*}$ for all $e \in E, f \in F$, where $e^{*}$ is a fixed element of $E$. For both semigroups we can show that every right ideal has the property stated in 3.6. All the right ideals in the latter semigroup $T$ are listed according to the chain $T \supset f T \supset e^{*} T \supset 0$, where $f \in F, e^{*} T=E \cup 0$ and $f T=E \cup F \cup 0$. The idempotent generator of $e^{*} T$ which commutes with all elements not in this ideal is the idempotent $e^{*}$.

We now give an example of an idempotent semigroup $S$ in which every right ideal is generated by an idempotent, but such that $S$ is not completely right injective. Let $S=\left\{0,1, e_{1}, e_{2}, f_{1}, f_{2}\right\}$ where 0 and 1 are the zero and identity elements of $S$, respectively. Define

$$
\begin{aligned}
& e_{i} e_{j}=e_{j}, \quad f_{i} f_{j}=f_{j}, \quad f_{i} e_{j}=e_{j} \quad(i, j=1,2), \\
& e_{1} f_{1}=e_{1}, \quad e_{1} f_{2}=e_{2}, \quad e_{2} f_{1}=e_{1}, \quad e_{2} f_{2}=e_{2} .
\end{aligned}
$$

Every right ideal of $S$ is generated by an idempotent; in fact, all the right ideals of $S$ can be exhibited in the chain $S \supset f_{i} S \supset e_{i} S \supset 0$. The right ideal $e_{i} S$ contains no idempotent which commutes with every $f_{j}$. By 3.6 , it follows that $S$ is not completely right injective.

\section{REFERENCES}

1. A. H. Clifford and G. B. Preston, The algebraic theory of semigroups, Amer. Math. Soc. Mathematical Surveys 7, Vol. I (Providence, R.I., 1961).

2. E. H. Feller and R. L. Gantos, Completely injective semigroups with central idempotents, Glasgow Math. J. 10 (1969), 16-20. 359-366.

3. E. H. Feller and R. L. Gantos, Completely injective semigroups, Pacific J. Math. 31 (1969),

UNIVERSITY OF WISCONSIN

MiLWAUKeE, U.S.A. 